\title{
Molecular dynamics and metadynamics simulations of electrosprayed water nanodroplets including sodium bis(2-ethylhexyl)sulfosuccinate micelles
}

Giovanna Longhi, Alberto Ceselli, Sandro L. Fornili, and Vincenzo Turco Liveri

Citation: The Journal of Chemical Physics 146, 204305 (2017);

View online: https://doi.org/10.1063/1.4984038

View Table of Contents: http://aip.scitation.org/toc/jcp/146/20

Published by the American Institute of Physics

\section{Articles you may be interested in}

Metadynamic metainference: Convergence towards force field independent structural ensembles of a disordered peptide

The Journal of Chemical Physics 146, 165102 (2017); 10.1063/1.4981211

Probabilistic inverse design for self-assembling materials

The Journal of Chemical Physics 146, 184103 (2017); 10.1063/1.4981796

Water structure around hydrophobic amino acid side chain analogs using different water models The Journal of Chemical Physics 146, 225104 (2017); 10.1063/1.4985671

Reducing the number of mean-square deviation calculations with floating close structure in metadynamics The Journal of Chemical Physics 146, 115101 (2017); 10.1063/1.4978296

IR spectral assignments for the hydrated excess proton in liquid water The Journal of Chemical Physics 146, 154507 (2017); 10.1063/1.4980121

Perspective: Thermal and thermoelectric transport in molecular junctions

The Journal of Chemical Physics 146, 092201 (2017); 10.1063/1.4976982

\section{AIP | The Jounal of Chemical Physics}




\title{
Molecular dynamics and metadynamics simulations of electrosprayed water nanodroplets including sodium bis(2-ethylhexyl)sulfosuccinate micelles
}

\author{
Giovanna Longhi, ${ }^{1,2, a)}$ Alberto Ceselli, ${ }^{3}$ Sandro L. Fornili, ${ }^{3}$ and Vincenzo Turco Liveri ${ }^{4}$ \\ ${ }^{1}$ Dipartimento di Medicina Molecolare e Traslazionale, Università di Brescia, Viale Europa 11, \\ 25123 Brescia, Italy \\ ${ }^{2}$ CNISM, Consorzio Interuniversitario Scienze Fisiche della Materia, Via della Vasca Navale 84, \\ 00146 Roma, Italy \\ ${ }^{3}$ Dipartimento di Informatica, Università di Milano, Via Bramante 65, 26013 Crema, Cremona, Italy \\ ${ }^{4}$ Dipartimento di Scienze e Tecnologie Biologiche, Chimiche e Farmaceutiche "STEBICEF," \\ Università degli Studi di Palermo, Viale delle Scienze I, 90128 Palermo, Italy
}

(Received 2 March 2017; accepted 10 May 2017; published online 26 May 2017)

\begin{abstract}
The behavior of aqueous solutions of sodium bis(2-ethylhexyl)sulfosuccinate (AOTNa) under conditions of electrospray ionization (ESI) has been investigated by molecular dynamics (MD) and well-tempered metadynamics (WTM) simulations at $300 \mathrm{~K}$ and $400 \mathrm{~K}$. We have examined water droplets with initial fixed numbers of water molecules (1000) and AOT $^{-}$anions (100), and with sodium cations in the range of 70-130. At $300 \mathrm{~K}$, all charged droplets show the water evaporation rate increasing with the absolute value of the initial droplet charge state $(\mathrm{Z})$, accompanied by ejection of an increasing number of solvated sodium ions or by expulsion of $\mathrm{AOT}^{-}$anions depending on the sign of $\mathrm{Z}$ and by fragmentation in the case of high IZl. At $400 \mathrm{~K}$, the water evaporation becomes more rapid and the fission process more extensive. In all cases, the AOTNa molecules, arranged as a direct micelle inside the aqueous system, undergo a rapid inversion in vacuo so that the hydrophilic heads and sodium ions surrounded by water molecules move toward the droplet interior. At the end of the 100-ns MD simulations, some water molecules remain within the aggregates at both temperatures. The subsequent metadynamics simulations accelerate the droplet evolution and show that all systems become anhydrous, in agreement with the experimental results of ESI mass spectrometry. This complete water loss is accompanied by sodium counterion emission for positively charged aggregates at $300 \mathrm{~K}$. The analysis shows how the temperature and droplet charge state affect the populations of the generated surfactant aggregates, providing information potentially useful in designing future ESI experimental conditions. Published by AIP Publishing. [http://dx.doi.org/10.1063/1.4984038]
\end{abstract}

\section{INTRODUCTION}

Electrospray ionization (ESI) is a technique extensively employed in mass spectrometry (MS) to produce beams of a wide variety of charged species. Its main merit is the ability to generate ions with minimal or no molecular decomposition, thanks to the low energy input necessary for their injection into the gas phase and subsequent passage to the vacuum stages of the mass spectrometer. For this reason, ESI is also a wellsuited technique for the production of charged non-covalent supramolecular aggregates in the gas phase.

The operating principle is quite simple: a solution, delivered through a metallic capillary connected to a high voltage (usually $2-5 \mathrm{kV}$ ), is ejected from the Taylor cone as micrometer-sized and highly charged droplets into a gas bath. ${ }^{1,2}$ Triggered by the gas temperature and cone voltage, ${ }^{3}$ the volatile components of the droplets evaporate leading to a decrease of the droplet size and consequently an increase of the non-volatile component concentration and the droplet

\footnotetext{
a) Author to whom correspondence should be addressed: giovanna.longhi@ unibs.it
}

charge density. Above a threshold value, the Coulomb repulsion causes the droplet explosion and/or solvated ion emission. Thus, the charged species observed by mass spectrometry are generated through a cascade of evaporation/fission/emission events.

The production of small ions is considered to be mainly driven by their emission from nanometer-sized droplets due to electrostatic repulsion forces, according to the ion emission model (IEM), whereas large globular species are believed to be formed by complete evaporation of volatile components in agreement with the charge residue model (CRM). ${ }^{4,5}$ However, these two models do not encompass all the possible mechanisms leading to the formation of charged species, so that hybrid and chain ejection models have been also proposed. ${ }^{6,7}$ Moreover, it becomes increasingly evident that the formation mechanism and the relative abundances of charged species in the gas phase should be system-specific and influenced by ESI conditions (temperature, capillary polarity, cone voltage). This is particularly true for non-covalent supramolecular aggregates, such as organized assemblies of ionic surfactants, because the coexistence of small-size counterions and relatively large surfactant ions does not allow to predict the 
mechanism of their formation in the gas phase and how it is affected by ESI conditions.

Considering that the control of the formation and of the relative amounts of surfactant aggregate populations in vacuo are of interest to estimate their potential technological and biotechnological applications (e.g., atmospheric cleaning agents, carriers of pulmonary drugs, biomolecule protectors, specialized nanoreactors), here we report the results of a molecular dynamics (MD) and well-tempered metadynamics (WTM) study of the transfer from the aqueous solution to vacuum of aggregates formed by the paradigmatic ionic surfactant sodium bis(2-ethylhexyl)sulfosuccinate (AOTNa). AOTNa is a well-known di-chained surfactant (see Fig. 1 of the supplementary material) able to form direct micelles in water above its critical micellar concentration (CMC), which is in the range $2.5-6 \cdot 10^{-3} \mathrm{M} .{ }^{8}$ In particular, we studied droplets composed of a fixed number of water molecules (1000) and AOT $^{-}$anions (100) and number of sodium cations ranging from 70 to 130. In such conditions, the surfactant concentration is about $6 \mathrm{~m}$ and the droplets can be considered as representative of those in the proximity of their terminal evaporation/fission/emission stages. $^{9-11}$

We will show that MD and WTM simulation results allow us to highlight the influence of temperature and droplet charge on the short-time (0-100 ns) evolution of the surfactant containing water droplets and the eventual state of the surfactant aggregates, thus highlighting the peculiar mechanism through which charged surfactant aggregates are formed by electrospray.

\section{COMPUTATIONAL METHODS}

The R-R-R diastereoisomer of AOT ${ }^{-}$(Fig. 1 of the supplementary material) has been modelled according to the all-atom general amber force field (GAFF), ${ }^{12}$ as previously reported. ${ }^{13}$

All simulations were done using the GROMACS-5.0.4 molecular dynamics package. ${ }^{14}$ The LINCS algorithm ${ }^{15}$ was used to constrain all bonds. The temperature was controlled by the velocity rescaling approach. ${ }^{16}$ For the metadynamics simulations, ${ }^{17}$ GROMACS was patched with PLUMED-2.1.1. ${ }^{18}$

Preliminarily, an aqueous system consisting of ca. 28000 TIP3 $\mathrm{P}^{19}$ waters and $100 \mathrm{AOT}^{-} \mathrm{Na}^{+}$was prepared in an octahedral box with the Leap module of AmberTools ${ }^{20}$ and a MD simulation at $\mathrm{T}=300 \mathrm{~K}$ and $\mathrm{p}=1 \mathrm{bar}$ was conducted using the particle-mesh Ewald (PME) method ${ }^{17}$ and periodic boundary conditions (PBC). In this way, we induced the formation of a $100-\mathrm{AOT}^{-} \mathrm{Na}^{+}$direct micelle, i.e., an aggregate characterized by an internal core formed by the AOT $^{-}$alkyl chains and an external layer made by the surfactant heads and sodium counterions. Then, $\mathrm{Na}^{+}$ions and water molecules whose distance from AOT $^{-}$was larger than $3 \AA$ were removed, and the charge of each droplet was adjusted by adding an appropriate number of $\mathrm{Na}^{+}$ions by randomly replacing water molecules as indicated in Ref. 22. The final number of the water molecules was then adjusted to 1000 for each droplet. As an example, the initial configuration for the droplet with $130 \mathrm{Na}^{+}$ions is presented in Fig. 1.

Following the procedure described by Konermann and coworkers, ${ }^{5,22}$ each droplet was placed at the center of a cubic box

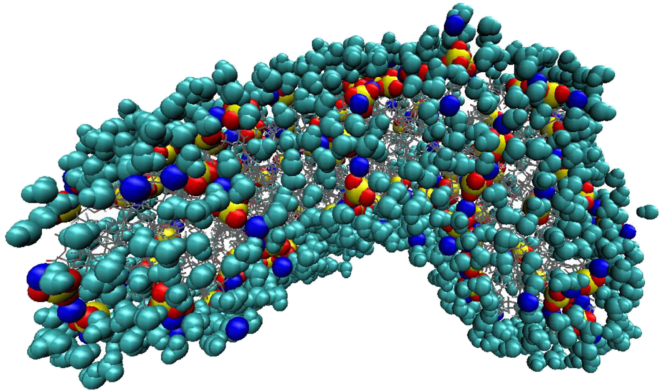

FIG. 1. Direct micelle of $100 \mathrm{AOT}^{-}$ions surrounded by a layer of 1000 water molecules and $130 \mathrm{Na}^{+}$ions (see text). The oxygen and the sulfur atoms of the $\mathrm{SO}_{3}{ }^{-}$polar heads of $\mathrm{AOT}^{-}$are displayed in red and yellow, respectively, and water molecules in light blue. Gray color indicates $\mathrm{AOT}^{-}$alkyl chains.

with side length of $999.9 \mathrm{~nm}$, and a cut-off value of $300 \mathrm{~nm}$ was assumed for the potential-shifted van der Waals and Coulomb interactions. This allowed to take advantage of the computational acceleration provided by the graphics processing unit (GPU), which requires PBC, while making negligible the interaction between replicas. In order to counteract the cooling of the droplet due to water evaporation, ${ }^{23,24}$ all velocities were randomly reassigned from a Maxwell distribution corresponding to the simulated temperature at the end of each 10-ps simulation interval. At the same time, the water molecules and $\mathrm{Na}^{+}$ions whose distance from each $\mathrm{AOT}^{-}$ion was larger than $20 \AA$ were removed.

The final configuration of each 100-ns MD simulation was taken to initiate the corresponding metadynamics simulation, during which all velocities were randomly reassigned after each 10-ps time interval as for MD simulations, but no water molecule was removed from the system. Each simulation lasted until the aggregate was found anhydrous. A bias potential was applied to the coordination number between the group of the $\mathrm{AOT}^{-} \mathrm{S}$ atoms and the group of the water oxygen atoms within a distance of $5 \AA$. After several trials, we chose the following WTM metadynamics parameters for the Gaussian height and bias factor, respectively: $10 \mathrm{~kJ} / \mathrm{mol}$ and 5000 at $300 \mathrm{~K}$, and $1 \mathrm{~kJ} / \mathrm{mol}$ and 100 at $400 \mathrm{~K}$. In all cases, we assumed a Gaussian deposition stride of 4 ps and a Gaussian width of 2.5, as evaluated according to the procedure outlined in Ref. 25.

The trajectory analysis for aggregate classification was performed with a home-made program exploiting graph theoretical tools, similarly to what was done in Ref. 26. In particular, the graph considers one vertex for each $\mathrm{Na}^{+}$ion, water oxygen atom, and $\mathrm{AOT}^{-} \mathrm{S}$ atom. Furthermore, one edge is included between each pair of vertices according to a matrix of parameters describing the interactions among water, sodium ions, and $\mathrm{AOT}^{-}$ions. Then, the graph is partitioned into subgraphs, representing aggregates in the MD simulation snapshot. Such partitioning is performed with a three-step clustering algorithm that (a) searches for connected components of ion and oxygen atom vertices, (b) assigns each water vertex to the nearest adjacent component, and, if any exists, (c) searches for connected components of unassigned water vertices. The search for connected components is carried out using the breadth-first-search algorithm. ${ }^{27}$ The program, coded in $\mathrm{C}++$, awk, and shell script, parses the 
trajectories and reports statistics on the aggregates number and composition.

Graphical analysis was performed using the Visual Molecular Dynamics (VMD) software. ${ }^{28}$

\section{RESULTS AND DISCUSSION}

As described in the section titled Computational methods, the initial configurations of MD simulations were direct micelles consisting of $100 \mathrm{AOT}^{-}$anions surrounded by 1000 water molecules (Fig. 1) and a suitable number of sodium ions to obtain charge defect or excess as reported in Table I. Hereafter, the aggregate composition will be indicated by the number of $\mathrm{AOT}^{-}$anions and sodium cations as $\left[\mathrm{N}_{\mathrm{AOT}^{-}}, \mathrm{N}_{\mathrm{Na}^{+}}\right]$ and its charge state by $\mathrm{Z}=\mathrm{N}_{\mathrm{Na}^{+}}-\mathrm{N}_{\mathrm{AOT}^{-}}$.

The main features of the behavior of these systems along the $100-\mathrm{ns}$ MD simulations in vacuo at $300 \mathrm{~K}$ or $400 \mathrm{~K}$ are summarized in Table I. It is worth to note that, at $300 \mathrm{~K}$, the negatively charged droplets with $\mathrm{Z}<-5$ undergo a more or less marked fragmentation process while the positively charged ones fragment when $\mathrm{Z}>10$ and release sodium ions when $\mathrm{Z}$ $>5$. This behavior highlights some differences in the stability of positively and negatively charged AOTNa aggregates and is consistent with the previous experimental and theoretical findings. ${ }^{29,30}$

On the other hand, irrespective of the droplet charge polarity, the number of the residual water molecules shows a tendency to decrease with the charge state (see Fig. 2 of the supplementary material). Besides, the water loss of negatively charged droplets is larger than that of the corresponding positively charged ones, the difference increasing with $|Z|$. Water evaporation is accompanied by ejection of an increasing number of sodium ions for positively charged droplets with $\mathrm{Z}>5$ and fragmentation when $\mathrm{Z}>10$ or by expulsion of $\mathrm{AOT}^{-}$ anions and aggregate fragmentation for negatively charged ones with $\mathrm{Z}<-5$. This suggests that sodium ions or aggregate fragments are ejected as solvated species, so promoting water evaporation. From Table I, one can see that after 100-ns

TABLE I. Main features of AOTNa-containing water droplets at the end of 100-ns MD simulations and subsequent WTM simulations. [N $\left.{ }^{0}{ }_{\text {AOT }}{ }^{-}, \mathrm{N}^{0}{ }_{\mathrm{Na}^{+}}\right]$: initial system composition; $\mathrm{n}\left[\mathrm{N}_{\mathrm{AOT}^{-}}, \mathrm{N}_{\mathrm{Na}^{+}}\right]$: $\mathrm{n}$ aggregates of composition $\left[\mathrm{N}_{\mathrm{AOT}^{-}}, \mathrm{N}_{\mathrm{Na}^{+}}\right]$; $\mathrm{Z}_{\mathrm{lf}}$ : charge of the largest aggregate; Nw: number of residual water molecules. Bold type evidences the largest aggregates.

\begin{tabular}{|c|c|c|c|c|c|}
\hline \multicolumn{4}{|c|}{ MD } & \multicolumn{2}{|l|}{ WTM } \\
\hline$\left[\mathrm{N}^{0}{ }_{\mathrm{AOT}^{-}}, \mathrm{N}^{0}{ }_{\mathrm{Na}^{+}}\right]$ & $\mathrm{n}\left[\mathrm{N}_{\mathrm{AOT}^{-}}, \mathrm{N}_{\mathrm{Na}^{+}}\right]$ & $Z_{\text {lf }}$ & $\mathrm{N}_{\mathrm{w}}$ & $\mathrm{n}\left[\mathrm{N}_{\mathrm{AOT}^{-}}, \mathrm{N}_{\mathrm{Na}^{+}}\right]$ & $Z_{\text {lf }}$ \\
\hline \multicolumn{6}{|c|}{$\mathrm{T}=300 \mathrm{~K}$} \\
\hline$[100,130]$ & $3[1,2], 2[3,4], \mathbf{1}[\mathbf{9 1}, \mathbf{9 8}]$ & 7 & 240 & $3[1,2], 2[3,4], \mathbf{1}[\mathbf{9 1 , 9 6}]$ & 5 \\
\hline$[100,120]$ & $2[1,2], \mathbf{1}[\mathbf{9 8 , 1 0 5}]$ & 7 & 259 & $2[1,2], \mathbf{1}[\mathbf{9 8 , 1 0 1}]$ & 3 \\
\hline$[100,110]$ & $1[100,107]$ & 7 & 344 & $1[100,104]$ & 4 \\
\hline$[100,105]$ & $1[100,105]$ & 5 & 633 & $1[100,103]$ & 3 \\
\hline$[100,103]$ & $1[100,103]$ & 3 & 661 & $1[100,101]$ & 1 \\
\hline$[100,100]$ & $1[100,100]$ & 0 & 763 & $1[100,100]$ & 0 \\
\hline$[100,97]$ & $1[100,97]$ & -3 & 671 & $1[100,97]$ & -3 \\
\hline$[100,95]$ & $1[100,95]$ & -5 & 581 & $1[100,95]$ & -5 \\
\hline$[100,90]$ & $2[1,0], \mathbf{1}[\mathbf{9 8 , 9 0}]$ & -8 & 191 & $2[1,0], \mathbf{1}[\mathbf{9 8 , 9 0}]$ & -8 \\
\hline$[100,80]$ & $\begin{array}{l}6[1,0], 1[3,1], 1[4,2], 1[28,25], \\
\mathbf{1}[\mathbf{5 9}, \mathbf{5 2}]\end{array}$ & -7 & 69 & $\begin{array}{l}6[1,0], 1[3,1], 1[4,2], 1[28,25], \\
\mathbf{1}[\mathbf{5 9}, \mathbf{5 2}]\end{array}$ & -7 \\
\hline$[100,70]$ & $\begin{array}{l}15[1,0], 2[2,1], 1[3,2], 1[5,4] \\
1[7,5], 1[32,28], \mathbf{1}[\mathbf{3 4 , 2 9}]\end{array}$ & -5 & 36 & $\begin{array}{l}15[1,0], 2[2,1], 1[3,2], 1[5,4], \\
1[7,5], 1[32,28], \mathbf{1}[\mathbf{3 4}, \mathbf{2 9}]\end{array}$ & -5 \\
\hline \multicolumn{6}{|c|}{$\mathrm{T}=400 \mathrm{~K}$} \\
\hline$[100,130]$ & $\begin{array}{l}3[1,2], 1[2,3], 1[3,4], 1[3,5] \\
\mathbf{1}[\mathbf{8 9 , 9 7}]\end{array}$ & 8 & 22 & $\begin{array}{l}3[1,2], 1[2,3], 1[3,4], 1[3,5], \\
\mathbf{1}[\mathbf{8 9 , 9 7 ]}\end{array}$ & 8 \\
\hline$[100,120](1)$ & $1[4,5], \mathbf{1}[\mathbf{9 6}, \mathbf{1 0 3}]$ & 7 & 12 & $1[4,5], \mathbf{1}[\mathbf{9 6}, \mathbf{1 0 3}]$ & 7 \\
\hline$[100,120](2)$ & $1[1,2], \mathbf{1}[\mathbf{9 9 , 1 0 6}]$ & 7 & 14 & $1[1,2], \mathbf{1}[\mathbf{9 9 , 1 0 6}]$ & 7 \\
\hline$[100,120](3)$ & $1[1,2], 1[33,36], \mathbf{1}[\mathbf{6 6}, 72]$ & 6 & 7 & $1[1,2], 1[33,36], \mathbf{1}[\mathbf{6 6}, 72]$ & 6 \\
\hline$[100,110]$ & $1[5,6], \mathbf{1}[\mathbf{9 5}, \mathbf{1 0 2}]$ & 7 & 14 & $1[5,6], \mathbf{1}[\mathbf{9 5}, \mathbf{1 0 2}]$ & 7 \\
\hline$[100,105]$ & $1[100,105]$ & 5 & 12 & $1[100,105]$ & 5 \\
\hline$[100,103]$ & $1[100,103]$ & 3 & 25 & $1[100,103]$ & 3 \\
\hline$[100,100]$ & $1[100,100]$ & 0 & 18 & $1[100,100]$ & 0 \\
\hline$[100,97]$ & $1[100,97]$ & -3 & 13 & $1[100,97]$ & -3 \\
\hline$[100,95]$ & $1[100,95]$ & -5 & 13 & $1[100,95]$ & -5 \\
\hline$[100,90]$ & $1[1,0], \mathbf{1}[\mathbf{9 9 , 9 0}]$ & -9 & 4 & $1[1,0], \mathbf{1}[\mathbf{9 9 , 9 0}]$ & -9 \\
\hline$[100,80]$ & $7[1,0], 1[2,1], 1[9,7], \mathbf{1}[\mathbf{8 2 , 7 2}]$ & -10 & 6 & $7[1,0], 1[2,1], 1[9,7], \mathbf{1}[\mathbf{8 2 , 7 2}]$ & -10 \\
\hline \multirow[t]{2}{*}[100,70]{} & $7[1,0], 6[2,1], 1[3,2], 1[4,3]$ & & & $7[1,0], 6[2,1], 1[3,2], 1[4,3]$ & \\
\hline & $\begin{array}{l}1[4,2], 1[5,3], 1[5,4], 1[6,5], \\
2[7,6], 1[8,7], 1[8,6], 1[10,8], \\
\mathbf{1}[\mathbf{1 4 , 1 2}]\end{array}$ & -2 & 11 & $\begin{array}{l}1[4,2], 1[5,3], 1[5,4], 1[6,5] \\
2[7,6], 1[8,7], 1[8,6] \\
1[10,8], \mathbf{1}[\mathbf{1 4}, \mathbf{1 2}]\end{array}$ & -2 \\
\hline
\end{tabular}


MD simulations at $300 \mathrm{~K}$, the maximum charge of the positive aggregates is 7 , while the excess of $\mathrm{AOT}^{-}$with respect to $\mathrm{Na}^{+}$ is slightly higher.

Raising the temperature to $400 \mathrm{~K}$, water evaporation is enhanced, so that the number of residual water molecules is minimal and nearly independent of the droplet charge value. Moreover, the degree of fragmentation of positively and negatively charged droplets at a high charge state is more marked. This shows that low temperature conditions favor the preservation of large supramolecular aggregates, as expected. Incidentally, it is worth to note that the fragmentation of charged droplets occurs through charge separation processes while the release of neutral surfactant molecules is never observed. At $400 \mathrm{~K}$, the maximum $\mathrm{Na}^{+}$excess is 8 for positive systems and fragmentation occurs quite easily for the negative ones: the most abundant fragments are single $\mathrm{AOT}^{-}$, i.e., $[1,0]$, but a variety of other fragment types are also produced. The maximum charge state of the largest fragment $\left(Z_{\mathrm{lf}}\right)$ is -10 . The variability of the number of fragments produced has been tested with three independent simulations for the $[100,120]$ system at $400 \mathrm{~K}$ (Table I).

To gain a deeper insight into the structural evolution of water-surfactant clusters, we show in Fig. 2 some snapshots taken at selected times from MD simulations at $300 \mathrm{~K}$ and $400 \mathrm{~K}$ of the systems with initial aggregate composition, $[100,120]$ and $[100,80]$. In addition to the features underlined above, it is worth to note that water evaporation occurs through the formation of thorns and that the AOTNa molecules, initially arranged as a direct micelle inside the water droplet, undergo a rapid inversion, so that the hydrophilic heads and sodium ions surrounded by water molecules are diverted toward the droplet interior. Therefore, in contrast with the simplistic view that electric charges should be located at the aggregate surface, the need to maximize electrostatic attractive interactions involves the clustering of charges in the interior of the aggregate, while the surfactant alkyl chains preferentially tend to form an external apolar layer coating the aggregate. Due to the

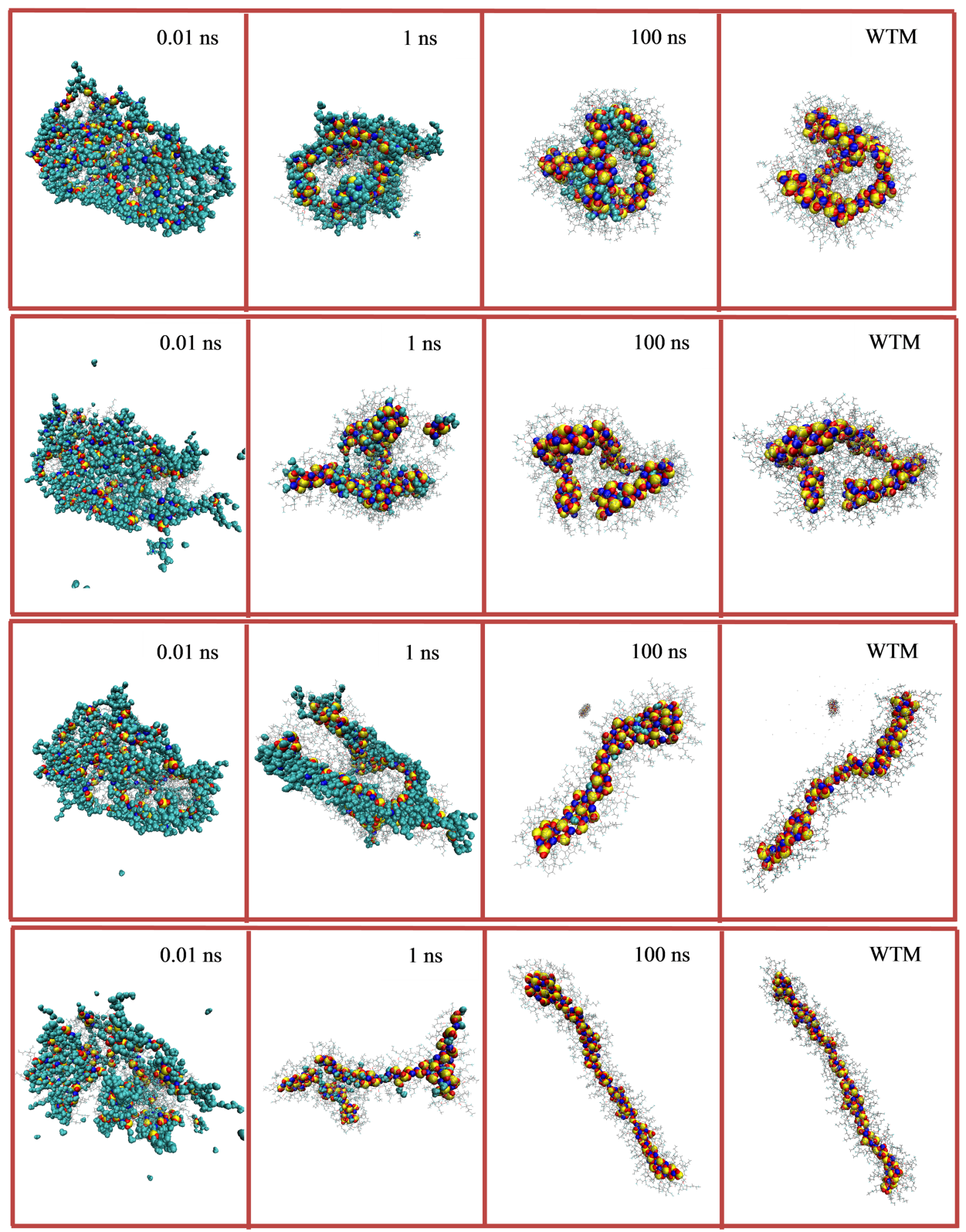

FIG. 2. Snapshots of surfactantcontaining water droplets at three MD simulation times $(0.01 \mathrm{~ns}, 1 \mathrm{~ns}$, and $100 \mathrm{~ns}$ ) and at the end of the WTM simulation. First and second row: $[100,120]$ system at $300 \mathrm{~K}$ and $400 \mathrm{~K}$, respectively. Third and fourth rows: $[100,80]$ system at $300 \mathrm{~K}$ and $400 \mathrm{~K}$, respectively. Sodium ions (blue), $\mathrm{SO}_{3}{ }^{-}$ polar heads (red oxygen atoms and yellow sulfur atoms), and water molecules (light blue) are displayed in a space-filling mode to show their location. AOT ${ }^{-}$alkyl tails are colored in gray. 

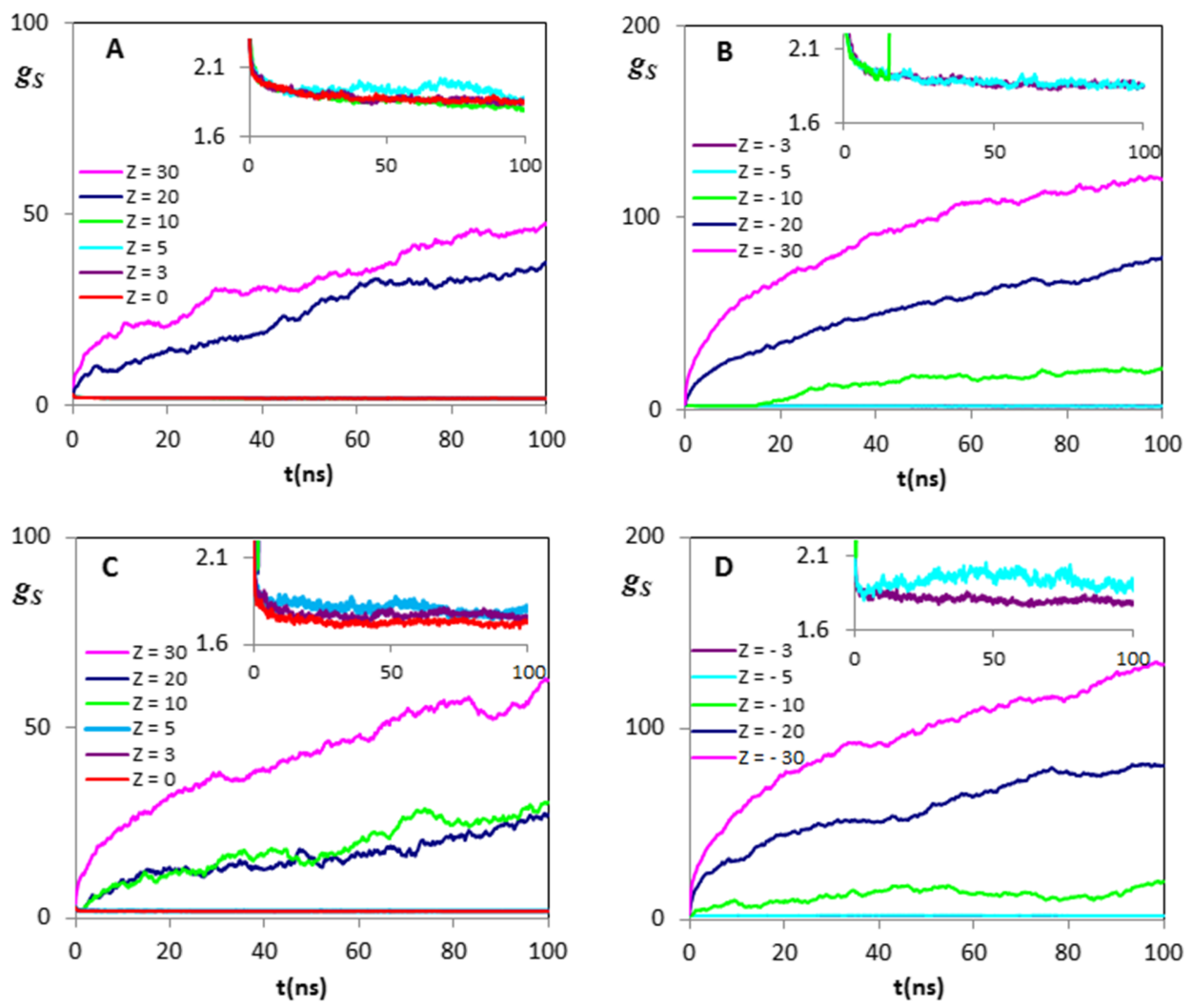

FIG. 3. Time dependence of gyration radius $\left(g_{S}\right)$ of sulfur atoms of the AOTNa head group at $300 \mathrm{~K}$ [panels (a) and (b)] and $400 \mathrm{~K}$ [panels (c) and (d)] at various charge values ( $Z$ ). Insets evidence the time evolution of cases with smaller $\boldsymbol{g}_{\boldsymbol{S}}$ values. high number of polar heads that cluster together, the shapes adopted by the aggregates upon drying are quite irregular, with a few of the alkyl chains secluded in the interior. ${ }^{31}$ The time scale of surfactant molecule inversion and/or fragmentation processes at $300 \mathrm{~K}$ and $400 \mathrm{~K}$ is evidenced in Fig. 3, where the gyration radius $\left(g_{S}\right)$ of sulfur atoms of the surfactant head group is reported as a function of time. Indeed, the rapid micelle inversion is revealed by the decrease of $\boldsymbol{g}_{\boldsymbol{S}}$ occurring mainly within the first ns: from Fig. 3 of the supplementary material one may observe that, at the very beginning, the gyration radius evaluated on sulfur atoms, $\boldsymbol{g}_{\boldsymbol{S}}$, has a higher value than the one evaluated on terminal C atoms (C9 and CI of Fig. 1 of the supplementary material), $\boldsymbol{g}_{\boldsymbol{C}}$, in a few hundreds of ps the opposite is observed. Considering longer times, in Fig. 3, we observe that large and fluctuating $\boldsymbol{g}_{\boldsymbol{S}}$ values are the signature of fragmentation processes that cause separation of $\mathrm{S}$ atoms. When fragmentation is present, a $\boldsymbol{g}_{\boldsymbol{S}}$ increase is observed nearly immediately after the simulation starts and this fact masks the decrease due to micelle inversion. This suggests that the initial intense evaporation and the surfactant rearrangement process cooperate to destabilize the aggregate enhancing the fragmentation probability. Not considering the cases undergoing fragmentation, final $g_{S}$ values are in the range of $1.7-1.9 \mathrm{~nm}$, indicating that system cores are quite compact and devoid of most of the water molecules. On the contrary, final gyration radii calculated on carbon C9 and CI (see Fig. 1 of the supplementary material), $g_{C}$, vary in the range of $2.25-2.6 \mathrm{~nm}$.

The time evolution of the number of the water molecules $(\mathrm{Nw})$, the system potential energy (PE), the number of sodium ions $\left(\mathrm{N}_{\mathrm{Na}^{+}}\right)$, and the number of fragments at $300 \mathrm{~K}$ for positively and negatively charged droplets are shown in Fig. 4. For comparison, the analogous data for a pure water droplet consisting of 1000 water molecules previously equilibrated with a MD simulation at $300 \mathrm{~K}$ and 1-bar pressure are also reported in (a) and (b) panels. As shown in Figs. 4(a) and 4(b), the initial water evaporation rate from the AOTNa-containing droplets increases with the charge state, while at longer times, the evaporation rate decreases and all the curves show a similar slope. We can think that the observed enhancement of water loss is assisted by the rearrangement of the surfactant molecules within the droplet, the ion emission, and/or the aggregate fragmentation. Indeed, these processes occur mainly in the early stage of MD simulations, as it has been already pointed out. It is worth to note that the behavior of the pure water droplet is characterized by an almost linear trend, meaning that the evaporation rate is nearly constant and similar to that initially shown by the surfactant-containing droplets at a low charge state.

As to the sodium ion emission, it can be noted that only the positively charged droplets with $\mathrm{Z}>5$ eject sodium ions and this occurs stepwise in the first part of the simulations (Fig. 4(c)). Moreover, consistent with a previous study, ${ }^{32}$ the data for the largest fragments reported in Table I indicate that at $300 \mathrm{~K}$ aggregates can stably held up to 7 excess sodium ions. On the other hand, the MD of negatively charged droplets with $\mathrm{Z}<-5$ is characterized by a fragment abundance which becomes increasingly conspicuous with the initial charge state of the droplets (Fig. 4(d)). Interestingly, sometimes fragments coalesce for a while during the simulation even if they have the same charge polarity. This behavior is in contrast with that of sodium ions emitted from positively charged droplets which never recapture them. 

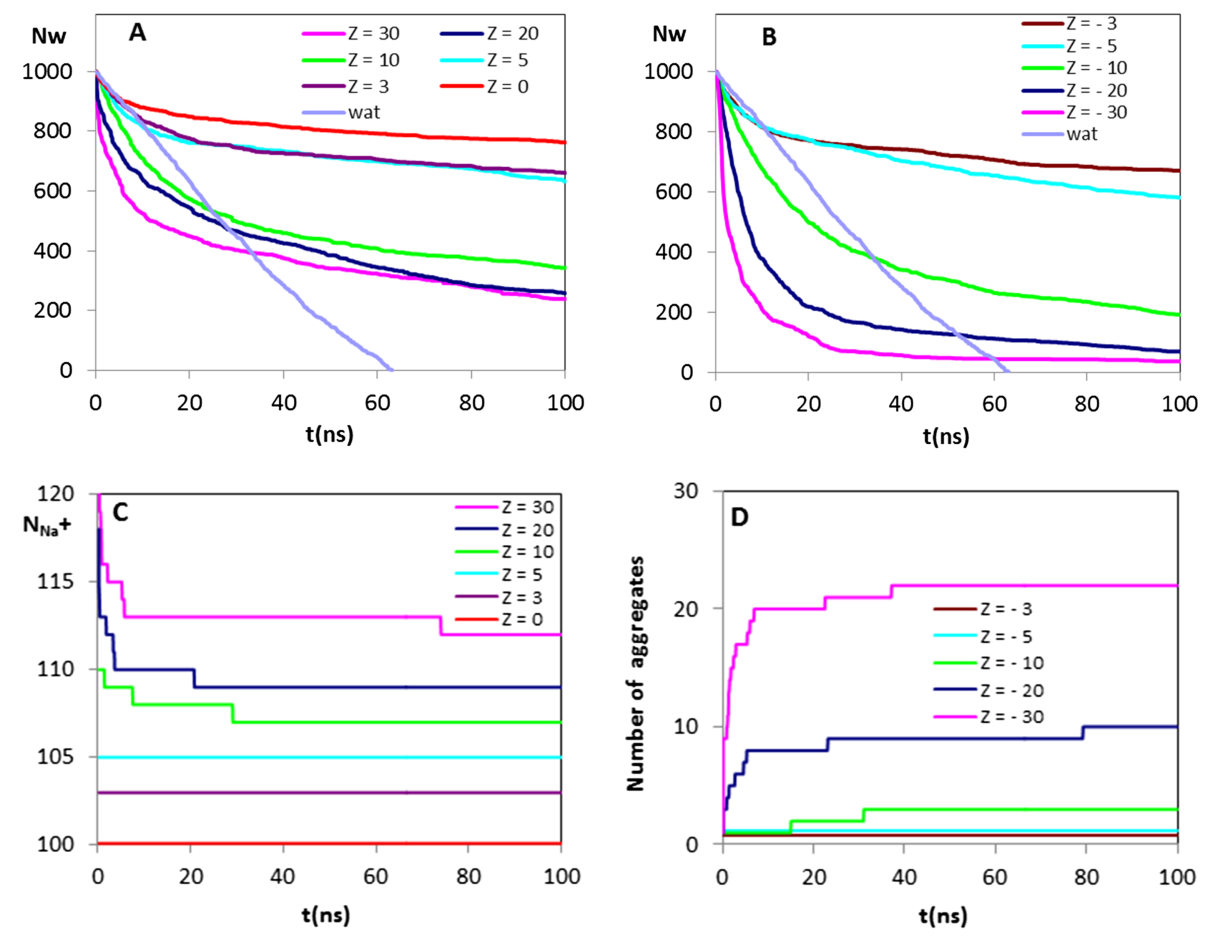

FIG. 4. Time evolution of the principal characteristics of positively (left panels) and negatively charged (right panels) droplets at $300 \mathrm{~K}$. (a) and (b): numbers of water molecules (Nw), (c): number of sodium ions $\left(\mathrm{N}_{\mathrm{Na}^{+}}\right)$, (d): number of fragments, and (e) and (f): system potential energy (PE) In panels (a) and (b), the $\mathrm{Nw}$ vs $\mathrm{t}$ curves of the neutral AOTNa-containing droplet and pure water droplet are also reported.
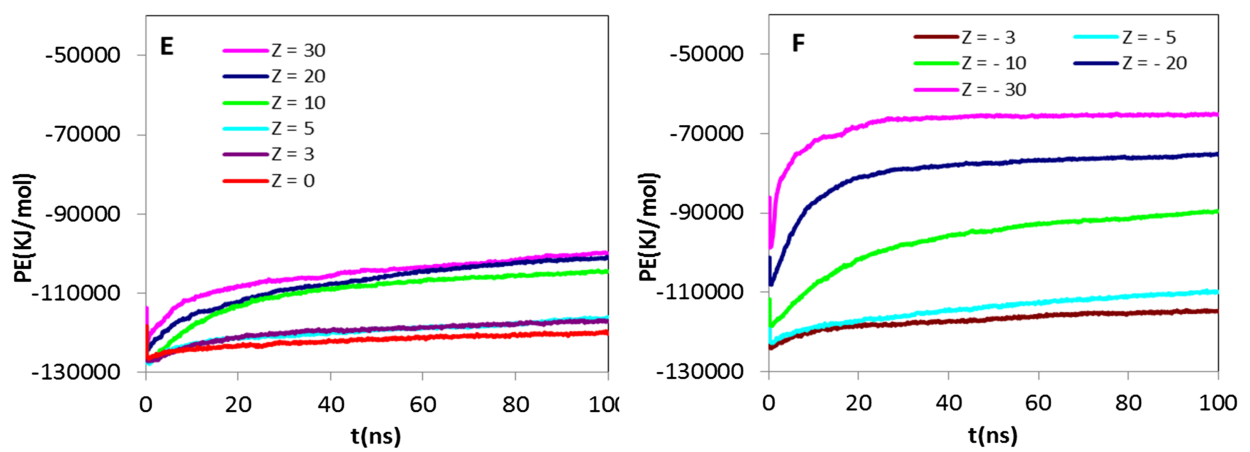

Both water evaporation and ion emission/fragmentation processes are also monitored by the PE vs. time curves [Figs. 4(e) and 4(f)]. Indeed, it can be noted an initial rapid PE decrease, attributable to a fast rearrangement of sodium ions and surfactant head groups occurring in a time interval of about $50 \mathrm{ps}$, followed by an increase due to water evaporation. Instead, ion emission/fragmentation processes are expected to give a negative contribution to PE due to the Coulomb repulsion term. Thus, the PE trends appear to be correlated with the number of water molecules evaporated and ions/fragments emitted.

Analogous time evolution curves for MD simulations at $400 \mathrm{~K}$ are shown in Fig. 5. It is evident that at $400 \mathrm{~K}$, the rate of water expulsion from the AOTNa-containing droplets is markedly higher than that at $300 \mathrm{~K}$, leaving the aggregates at the end of MD simulations with a lower number of water molecules. It can be noted, however, that at longer times, while the pure water droplet evaporates completely, a residual number of water molecules remain tightly bonded to surfactant head groups and sodium counterions.

The sodium ion ejection from positively charged droplets is slightly affected by the temperature increase; in fact, at $400 \mathrm{~K}$, the maximum excess number of sodium ions is 8 . As to the negatively charged droplets, the loss of sodium ions is absent, as expected, but they feature a fragmentation degree more pronounced than for the positively charged ones (see Table I and Fig. 4(d)). All these effects are also monitored by the PE trends shown in Figs. 5(e) and 5(f). In particular, the abundant loss of water molecules and the marked fragmentation imply an increase of PE at $400 \mathrm{~K}$ larger than that at $300 \mathrm{~K}$.

\section{METADYNAMICS SIMULATIONS}

Taking into account that the behavior of surfactantcontaining water droplets has been observed with MD simulations in vacuo within a time window of $100 \mathrm{~ns}$, while the time scale of mass spectrometry experiments is typically in the $\mu \mathrm{s}-\mathrm{ms}$ range, ${ }^{29}$ an enlargement of the time window seems indispensable. Since this appears practically infeasible with MD due to the large computer time requirement, we turned to metadynamics simulations. ${ }^{17}$ This method, in fact, drives the system evolution by means of a biasing potential constructed as a sum of Gaussians allowing to explore the space of appropriately chosen collective variable(s). In this way, the system is forced to visit conformations, overshooting relatively high 

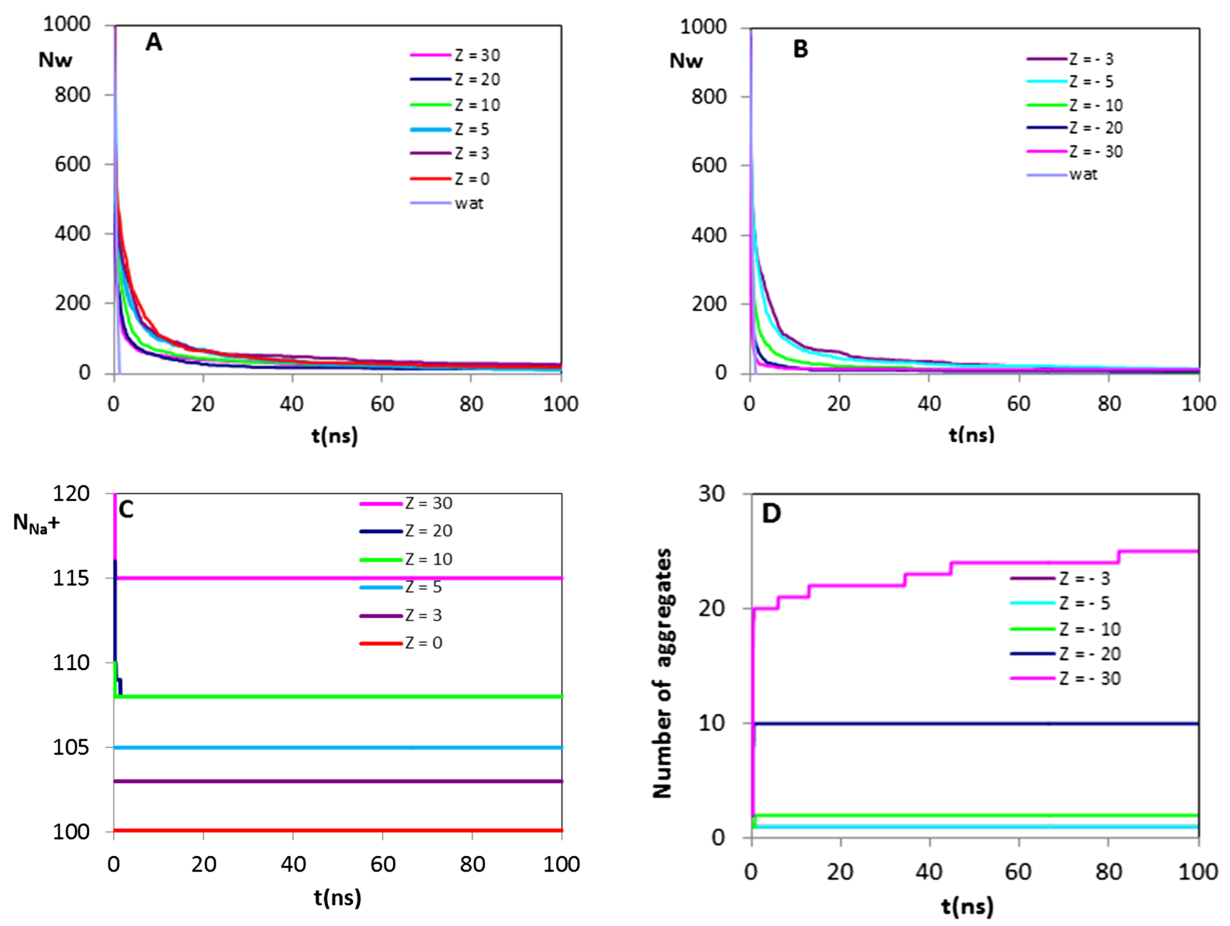

FIG. 5. Time evolution of the principal characteristics of positively (left panels) and negatively charged (right panels) droplets at $400 \mathrm{~K}$. (a) and (b): numbers of water molecule $(\mathrm{Nw})$, (c): number of sodium ions $\left(\mathrm{N}_{\mathrm{Na}^{+}}\right)$, (d): number of fragments, and [(e) and (f)]: system potential energy (PE). In panels (a) and (b), Nw vs t curves of the pure water droplet and of the neutral AOTNa-containing droplet are also reported.
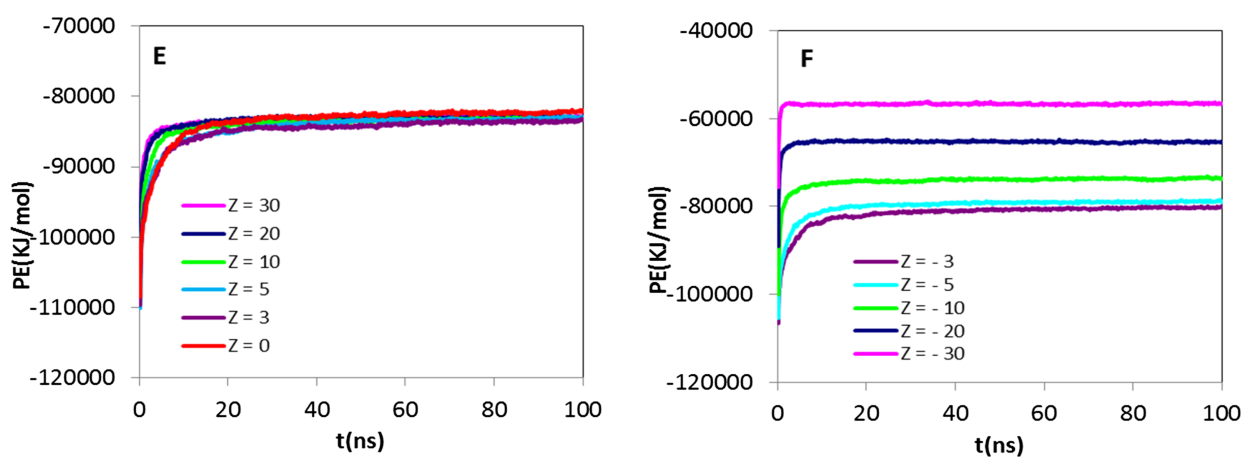

energy barriers. Moreover, it provides an estimate of the system free energy (FE) as a function the collective variable(s), thus allowing to find equilibrium conformations corresponding to the FE global minimum. As an appropriate collective variable, we have chosen the coordination number $(\mathrm{CN})$ between the group of $\mathrm{AOT}^{-} \mathrm{S}$ atoms and the group of the water oxygen
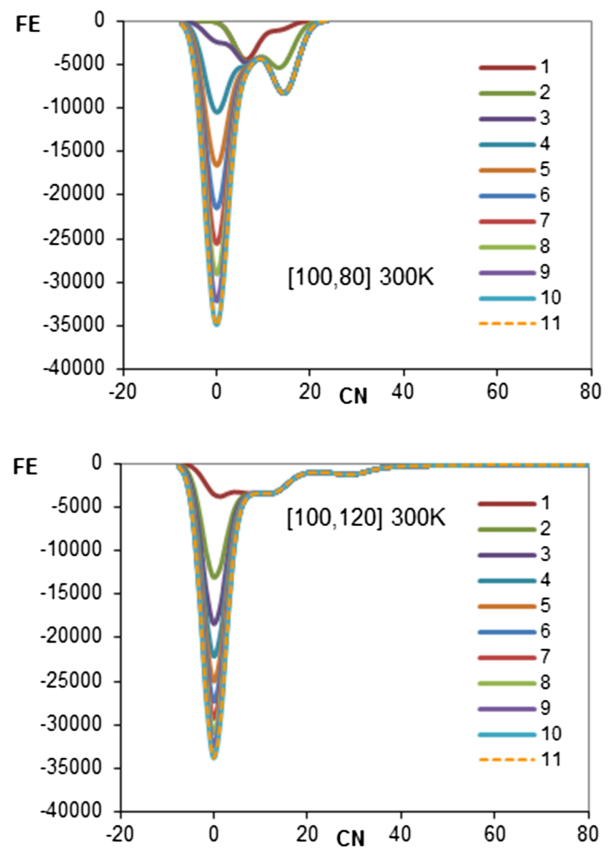
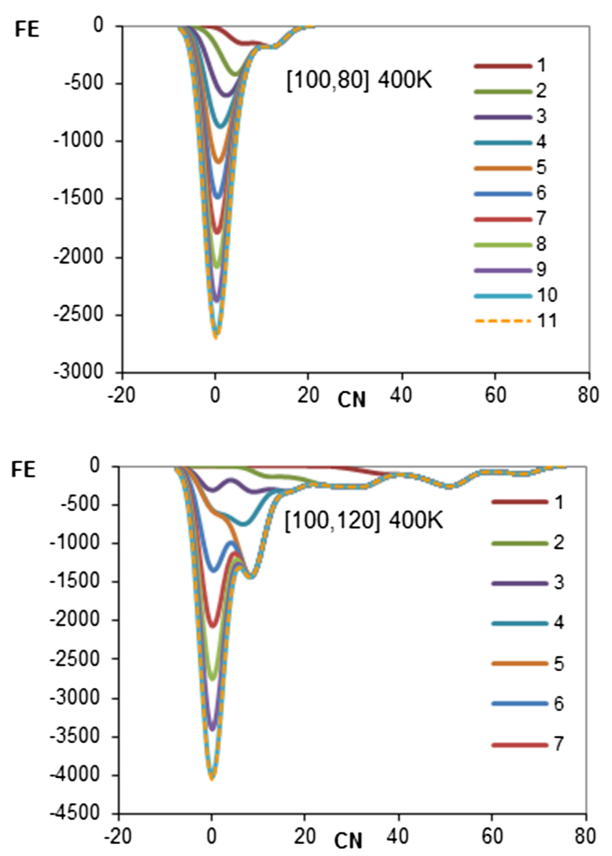

FIG. 6. Free energy (FE) vs coordination number $(\mathrm{CN})$ between the AOT $^{-}$ $\mathrm{S}$ atoms and the water $\mathrm{O}$ atoms. The curves are evaluated through equispaced sampling of the WT metadynamics trajectories. 
atoms, as outlined in the section titled Computational methods. Figure 6 shows some representative curves of FE vs CN for WTM simulations at $300 \mathrm{~K}$ and $400 \mathrm{~K}$. It is worth to note that in all cases, the FE global minimum occurs at $\mathrm{CN}$ $=0$. Thus, the most important result obtained by using the WTM simulation approach is that eventually all the examined AOTNa-water systems completely release water even at $300 \mathrm{~K}$, in full agreement with experimental findings. ${ }^{11}$ Moreover, a perusal of the last two columns of Table I shows that at the end of metadynamics experiments in addition to the complete loss of residual water molecules some changes in the aggregate charge state sometimes occur. It is worth to note that our results are obtained by applying the metadynamics approach to the study of the unidirectional long time evolution of molecular systems.

\section{CONCLUSIONS}

Molecular dynamics (MD) and well-tempered metadynamics (WTM) simulations have been employed to study the behavior in vacuo of water droplets containing the paradigmatic ionic surfactant sodium bis(2-ethylhexyl)sulfosuccinate (AOTNa) under electrospray ionization (ESI) conditions. The analysis of MD and WTM trajectories allow us to emphasize the peculiar mechanisms through which surfactant aggregates observed by mass spectrometry are formed. In particular, the current models for the production of charged species by electrospray, IEM and CRM, appear inadequate to describe the time evolution of charged surfactant-containing water droplets. Actually, the present data indicate that the temperature, charge state, and polarity of the droplet affect so strongly the involved molecular processes and the populations of the produced AOTNa aggregates, that it seems quite implausible the adoption of a simple modeling scheme.

Together with water evaporation and a rapid inversion of surfactant molecules orientation within the droplet, sodium ion and $\mathrm{AOT}^{-}$ejection and aggregate fragmentation occur, as triggered by the temperature and droplet charge. Thus, by changing these parameters, the produced aggregate populations shift from those foreseen by the CRM to a fragment emission model involving the production of a range of charged aggregates. More generally, we propose that the ion production model for supramolecular noncovalent aggregates formed by surfactant molecules should be system-specific and strongly dependent on the experimental conditions.

MD and WTM simulations allowed also to highlight some peculiar features of the investigated systems. Interestingly, the water evaporation rate is significantly enhanced by the presence of surfactant molecules in the very first stages, when micelle inversion and high expulsion of ions occur; it increases with the droplet charge state while the droplet fission is boosted by an increase of the temperature and charge state. Moreover, in agreement with the experimental findings, the negatively charged droplets are found to be more prone to fragment than the positively charged ones, so emphasizing the role of the electrostatic interactions as determining factors of the aggregate stability. Considering the typical time scale of mass spectrometry experiments, the surfactant assemblies are expected to be found anhydrous, as it has been found in the present simulation procedure.

Overall, the thorough analysis of the MD and WTM simulation data enlighten the interesting and intricate behavior of charged AOTNa-containing water droplets under electrospray conditions, providing information potentially useful to select appropriately ESI experimental conditions for the production of charged supramolecular non-covalent aggregates and their detection by mass spectrometry.

\section{SUPPLEMENTARY MATERIAL}

See supplementary material for AOT atom numbering, plot of the number of water molecules at the end of 100-ns MD simulations as a function of the initial droplet charge, and plot of the time dependence of the gyration radii $g_{S}$ of the AOT $^{-}$sulfur atoms and $g_{C}$ of the terminal carbon atoms along the first $1.5 \mathrm{~ns}$ of MD simulations at $300 \mathrm{~K}$ and $400 \mathrm{~K}$.

\section{ACKNOWLEDGMENTS}

We thank the Computing Center CINECA via Magnanelli 6/3 40033 - Casalecchio di Reno (Bologna) Italy and Regione Lombardia for the LISA Grant No. "ChiPhyto: HPL13POZE1" for access to their computational facilities.

${ }^{1}$ M. Yamashitat and J. B. Fenn, J. Phys. Chem. 88, 4451 (1984)

${ }^{2}$ P. Pan and S. A. McLuckey, Anal. Chem. 75, 1491 (2003).

${ }^{3}$ V. A. Pashynska, M. V. Kosevich, H. Van den Heuvel, and M. Claeys, Rapid Commun. Mass Spectrom. 20, 755 (2006).

${ }^{4}$ P. Kebarle and U. H. Verkerk, Mass Spectrom. Rev. 28, 898 (2009).

${ }^{5}$ L. Konermann, R. G. McAllister, and H. Metwally, J. Phys. Chem. B 118, 12025 (2014).

${ }^{6}$ C. J. Hogan, J. A. Carroll, H. W. Rohrs, P. Biswas, and M. L. Gross, Anal. Chem. 81, 369 (2009).

${ }^{7}$ C. Larriba, F. de la Mora, and D. E. Clemmer, J. Am. Soc. Mass Spectrom. 25, 1332 (2014)

${ }^{8}$ F. Heatley, J. Chem. Soc., Faraday Trans. 1 83, 517 (1987).

${ }^{9}$ A. Wortmann, A. Kistler-Momotova, R. Zenobi, M. C. Heine, O. Wilhelm, and S. E. Pratsinis, J. Am. Soc. Mass Spectrom. 18, 385 (2007).

${ }^{10}$ P. Kebarle and L. Tang, Anal. Chem. 65, 972A (1993).

${ }^{11}$ D. Bongiorno, L. Ceraulo, A. Ruggirello, V. Turco Liveri, E. Basso, R. Seraglia, and P. Traldi, J. Mass Spectrom. 40, 1618 (2005).

${ }^{12}$ J. Wang, R. M. Wolf, J. W. Caldwell, P. A. Kollman, and D. A. Case, J. Comput. Chem. 25, 1157 (2004).

${ }^{13}$ G. Longhi, S. L. Fornili, V. Turco Liveri, S. Abbate, D. Rebeccani, L. Ceraulo, and F. Gangemi, Phys. Chem. Chem. Phys. 12, 4694 (2010).

${ }^{14}$ M. J. Abraham, D. van der Spoel, E. Lindahl, B. Hess, and GROMACS Development Team, GROMACS User Manual version 5.0.4, 2014, www.gromacs.org.

${ }^{15}$ B. Hess, J. Chem. Theory Comput. 4, 116 (2007).

${ }^{16}$ G. Bussi, D. Donadio, and M. Parrinello, J. Chem. Phys. 126, 014101 (2007).

${ }^{17}$ A. Barducci, G. Bussi, and M. Parrinello, Phys. Rev. Lett. 100, 020603 (2008).

${ }^{18}$ G. A. Tribello, M. Bonomi, D. Branduardi, C. Camilloni, and G. Bussi, Comput. Phys. Commun. 185, 604 (2014).

${ }^{19}$ W. L. Jorgensen, J. Chandrasekhar, J. D. Madura, R. W. Impey, and M. L. Klein, J. Chem. Phys. 79, 926 (1983).

${ }^{20}$ D. A. Case, V. Babin, J. T. Berryman, R. M. Betz, Q. Cai, D. S. Cerutti, T. E. Cheatham III, T. A. Darden, R. E. Duke, H. Gohlke, A. W. Goetz, S. Gusarov, N. Homeyer, P. Janowski, J. Kaus, L. Kolossvary, A. Kovalenko, T. S. Lee, S. LeGrand, T. Luchko, R. Luo, B. Madej, K. M. Merz, F. Paesani, D. R. Roe, A. Roitberg, C. Sagui, R. Salomon-Ferrer, G. Seabra, C. L. Simmerling, W. Smith, J. Swails, R. C. Walker, J. Wang, R. M. Wolf, X. Wu, and P. A. Kollman, AMBER 14, University of California, San Francisco, 2014.

${ }^{21}$ T. Darden, D. York, and L. Pedersen, J. Chem. Phys. 98, 10089 (1993). 
${ }^{22}$ R. G. McAllister, H. Metwally, Y. Sun, and L. Konermann, J. Am. Chem. Soc. 137, 12667 (2015).

${ }^{23}$ C. Caleman and D. van der Spoel, J. Chem. Phys. 125, 154508 (2006).

${ }^{24}$ C. Caleman and D. van der Spoel, Phys. Chem. Chem. Phys. 9, 5105 (2007).

${ }^{25}$ A. Laio and F. L. Gervasio, Rep. Prog. Phys. 71, 126601 (2008).

${ }^{26}$ G. Longhi, A. Ceselli, S. L. Fornili, S. Abbate, L. Ceraulo, and V. Turco Liveri, J. Mass Spectrom. 48, 478 (2013).

${ }^{27}$ D. E. Knuth, The Art of Computer Programming (Addison-Wesley, Boston, 1997), Vol. 1
${ }^{28}$ W. Humphrey, A. Dalke, and K. Schulten, J. Mol. Graphics 14, 33 (1996).

${ }^{29}$ S. Indelicato, D. Bongiorno, L. Ceraulo, V. Calabrese, D. Piazzese, A. Napoli, F. Mazzotti, G. Avellone, V. Di Stefano, and V. Turco Liveri, Rapid Commun. Mass Spectrom. 30, 230 (2016).

${ }^{30}$ G. Longhi, S. Abbate, A. Ceselli, L. Ceraulo, S. L. Fornili, and V. Turco Liveri, J. Am. Soc. Mass Spectrom. 25, 1642 (2014).

${ }^{31}$ G. Longhi, S. L. Fornili, and V. Turco Liveri, Phys. Chem. Chem. Phys. 17, 16512 (2015).

${ }^{32}$ G. Longhi, S. Abbate, L. Ceraulo, A. Ceselli, S. L. Fornili, and V. Turco Liveri, Phys. Chem. Chem. Phys. 13, 21423 (2011). 